\title{
Perception onset time during fixations in free viewing
}

\author{
GEORGE W. MCCONKIE and LESTER C. LOSCHKY \\ University of Illinois at Urbana-Champaign, Urbana, Illinois
}

\begin{abstract}
In this study, we investigated when visual perception begins in fixations. During picture viewing, the picture was degraded at the beginning of selected saccades and changed back to the original after varying intervals. Participants manually responded whenever they detected changes. The change-backs were undetected when they occurred $<6 \mathrm{msec}$ after the end of the saccade, marked by the peak of the overshoot in dual Purkinje image eyetracker data, and detection reached asymptote 32 msec after that marker. Eye velocity at the change-back time also affected detection likelihood. Apparently, perception begins around the time at which the eyes stop rotating at the end of a saccade, giving a psychological justification for measuring fixation durations from then. This also specifies the deadline for gazecontingent display changes to occur without detectable image motion. Investigators using the dual Purkinje image eyetracker should consider the peak of the overshoot as the fixation onset time and measure intrafixational presentation times from then.
\end{abstract}

People examine pictures by making a series of eye fixations, each with the gaze directed toward a different location, providing an opportunity to gain additional information from the picture. Visual input is largely suppressed during the saccadic eye movement that separates each pair of fixations because of a combination of factors, including the rapid motion of the light pattern across the retinal receptors (Brooks, Impelman, \& Lum, 1981; Chekaluk \& Llewellyn, 1990, 1994; Diamond, Ross, $\&$ Morrone, 2000) and some degree of suppression within the central nervous system itself (Burr \& Morrone, 1996; Burr, Morrone, \& Ross, 1994; Diamond et al., 2000; Paus, Marrett, Worsley, \& Evans, 1995; Uchikawa \& Sato, 1995). This being the case, at the end of each saccade, there must be some point at which the new stimulus pattern begins to be resolved. We will refer to this as the perceptual onset time. The goal of the study described below was to begin to identify when this occurs.

There are several reasons to be interested in the perceptual onset time. First, many researchers use fixation duration as a dependent variable in their work (see, e.g., Hayhoe, Bensinger, \& Ballard, 1998; Morrison, 1984; Rayner,

This work was supported by funds from the Beckman Institute, the University of Illinois Research Board, and the U.S. Army Research Laboratory under the Federated Laboratory Program, Cooperative Agreement DAAL01-96-2-0003. The National Institute of Mental Health provided funds for purchasing the eye-tracking equipment, and the National Science Foundation provided other essential equipment under Grant CDA 96-24396. The authors acknowledge the work of Gary Wolverton, who developed the system and wrote the software that made this research possible. The authors also thank Ed Niu for providing the wavelet decomposition and reconstruction algorithm. Correspondence concerning this article should be addressed to G. W. McConkie, 2251 Beckman Institute for Advanced Science and Technology, 405 North Mathews, Urbana, IL 61801 (e-mail: gmcconk@uiuc.edu).
1997). There is currently no consensus among laboratories as to when, at the beginning of each fixation, to start the clock. From a psychological perspective, it would seem appropriate to begin this measurement from the moment that perception begins after each saccade, if we knew when this occurred.

Second, for some studies, it is necessary to present a stimulus pattern for a specified period of time on some or all eye fixations during a viewing task (Rayner, Inhoff, Morrison, Slowiaczek, \& Bertera, 1981; van Diepen, Ruelens, \& d'Ydewalle, 1999; van Diepen \& Wampers, 1998; van Diepen, Wampers, \& d'Ydewalle, 1995, 1998). Since visual input is suppressed during saccades, the simple presentation of the stimulus pattern for a given period of time does not necessarily result in its being perceived for that time. The effective stimulus period will be shorter than the actual stimulus presentation period, if the stimulus presentation overlaps with a period during which vision is suppressed. We must know the perceptual onset time in order to avoid this problem.

Third, for other studies, it is necessary to make changes in the stimulus during the period of saccadic suppression, so that the stimulus motion that necessarily accompanies such a change is not directly detectable by the participant (Currie, McConkie, Carlson-Radvansky, \& Irwin, 2000; Grimes, 1996; Hayhoe et al., 1998; Henderson \& Hollingworth, 1999; McConkie \& Currie, 1996; McConkie \& Loschky, 2000). A particular problem occurs when it is necessary to create a stimulus configuration on the new fixation that will be determined by the location of the eyes during that fixation (Bertera \& Rayner, 2000; Henderson, McClure, Pierce, \& Schrock, 1997; Loschky \& McConkie, 2000, 2002; Loschky, McConkie, Yang, \& Miller, 2002; McConkie \& Rayner, 1975; Rayner et al., 1981; van Diepen et al., 1999; van Diepen \& Wampers, 
1998; van Diepen et al., 1995, 1998). In this case, it is necessary to wait until late in the saccade to accurately anticipate the eyes' landing position, creating the possibility that the stimulus change may not occur until after the perceptual onset time, thus allowing the change itself to be perceived. In these studies, it is important to know when perception begins in order to be sure that the display change and any perceptible persistence resulting from it have been completed by the perceptual onset time.

It might seem that the obvious thing to do in such studies is simply to take the point at which the eyes stop after a saccade as the perceptual onset time. However, this solution is not so simple, for several reasons. First, the point at which the eyes stop is itself not clear. The eyes gradually slow to a stop at the end of a saccade, often with further drift, which sometimes appears to be vergence movement. There is not a clear break between these different types of motion, and we do not know whether saccadic suppression continues for part or all of these slower movements. Second, even if we could unambiguously determine when the saccadic motion ends, it still would not be certain that this is also the perceptual onset time. One possibility is that the eyes' speed drops low enough prior to stopping, with the retinal image being sufficiently stable, that perception of the patterned stimuli can begin. Alternatively, it is possible that since saccadic suppression continues for some time following the end of the saccade (Burr et al., 1994; Diamond et al., 2000; Matin, 1974; Volkmann, 1962; Volkmann, Riggs, White, \& Moore, 1978), perceptual onset time may be delayed beyond the actual completion of external eye motion.

Finally, in our research, we use the dual Purkinje image eyetracker, which gives very high spatial and temporal resolution (Crane \& Steele, 1978). However, Deubel and Bridgeman (1995) have shown that its representation of the dynamic movement of the eyes during a saccade is somewhat inaccurate, in comparison with the movement shown by the search coil method: (1) The onset of motion in the Purkinje image eyetracker signal is slightly delayed, (2) at the end of the saccade, the signal shows a large overshoot that is greatly amplified in relation to similar patterns in the search coil data, and (3) motion in the signal often continues for $20 \mathrm{msec}$ or more after the end of motion in the search coil data. These differences arise as a result of the methods used by these two devices for monitoring the eyes. The search coil (Collewijn, Van der Mark, \& Jansen, 1975) lies directly on the surface of the eye or embedded in a contact lens, thus providing an accurate indication of the motion of the eye's surface. The Purkinje image eyetracker, on the other hand, tracks infrared light reflected from the front surface of the cornea and the back surface of the lens (Crane \& Steele, 1978). Thus, its signal reflects the motion of internal structures of the eyes, as well as that of the surface. The large overshoot at the end of the saccade, for example, is assumed to reflect motion of the lens as the forces within the eyeball act on it as rotation ends (Deubel \& Bridgeman,
1995). Deubel and Bridgeman reported that the peak of this overshoot in the Purkinje signal occurs at about the same time as the search coil signal indicates the end of saccadic motion. Thus, the motion in the Purkinje image eyetracker signal after the peak of the overshoot appears to result from motion of internal eye structures. On the one hand, these characteristics of the Purkinje image eyetracker signal can be seen as artifacts that make it less accurate in identifying the beginnings and ends of saccades. On the other hand, if motion of the lens after the eye stops rotating still causes motion of the image on the retina, it is possible that the perceptual onset time could be delayed. In this case, the Purkinje image eyetracker could yield an estimate of the perceptual onset time that is more accurate than that provided by the search coil method. It is not clear that accurate identification of the time at which the external parts of the eye stop moving will provide a better landmark for identifying the perceptual onset time than will the time at which the internal parts of the eye finally settle down.

Shioiri (1993) addressed the issue of when perception begins on a fixation, using a method quite similar to that used in the study described below. At the end of each saccade, the picture on the computer screen was replaced by a blank screen or a degraded version of the picture, and then the original version returned to the screen after a specified interval. He reported that the average median fixation duration was increased at even his shortest interval, $25 \mathrm{msec}$, and so he concluded that perception begins at the time the eyes stop. However, that study included no condition in which changes in the display went undetected. The experiment presented here differed from that of Shioiri in several ways. First, we changed the image to a degraded version only during selected saccades, which allowed us to use detection frequency as a measure. This was not possible in the Shioiri study, in which the degraded image or blank screen was present on every saccade. However, Shioiri did indicate that participants were able to see the changes that took place, and in all experimental conditions they produced changes in the frequency distributions of fixation durations (cf. Reingold \& Stampe, 1999). Second, in the present study, the degraded image version was placed on the screen at the beginning of the saccade, rather than at the end, and was removed after different periods of time. This made it possible to examine the effects of having the original image reappear at periods of time that were earlier, relative to the end of the saccade, than the intervals used by Shioiri and ensured that the first change, from the undegraded image to its degraded version, was not directly perceived.

The present study was motivated by the initiation of a series of experiments involving intrasaccadic display changes (Loschky \& McConkie, 2000, 2002; Loschky et al., 2002). In these experiments, participants viewed pictures, which we refer to as gaze-contingent multiresolutional images, with degradation in the parafovea and periphery and a region of high resolution at the point of 
gaze during each eye fixation (Reingold, Loschky, McConkie, \& Stampe, in press). Thus, it was necessary to display a different version of the image on each fixation, contingent upon the point of gaze for that fixation. Because we did not want the participants to directly perceive the display changes taking place, we wanted each display change to be completed prior to the perceptual onset time. To accomplish this, it was necessary to identify the perceptual onset time for the types of stimulus materials we were planning to use in these experiments. The characteristics of the present study were guided by this requirement.

The study was conducted by creating two versions of each of a number of pictures, one a normal, high-resolution version, and the second a degraded version. The participants were presented with the normal version of each picture and were asked to examine the picture carefully in preparation for a memory task in which they would be asked to identify which pictures had been changed. As a secondary task, they were instructed to press a button whenever they noticed a flicker or brief change in the picture's resolution. On selected saccades, the high-resolution version was replaced by the low-resolution version as soon as the eyes began to move (referred to as the change), with the high-resolution version returning after one of five periods of time, ranging from 14 to $70 \mathrm{msec}$ (referred to as the change-back). Later, the detection data were analyzed as a function of the time of the change-back relative to the end of motion of the eyes, relative to the peak of the overshoot, and as a function of the velocity of the eyes at the time the change-back occurred. These analyses were used to demonstrate a failure to detect degradation of the image during saccades and to provide information about when the perceptual onset time occurs at the end of the saccade, as indicated by a rise in this detection frequency. It should be noted that this type of design does not have discrete trials in which a single event either does or does not occur, nor is there a concomitant a priori chance-level detection likelihood (e.g., 50\%). Thus, detection-likelihood comparisons must be made against the likelihood of detection that occurs for a control (no-change) condition and for cases in which changes occur during saccadic eye movements when perception is suppressed, rather than against an a priori detection likelihood threshold.

\section{METHOD}

\section{Participants}

Twelve university students were paid for participating in this study. All had normal, uncorrected vision.

\section{Stimuli}

The study included 36 monochrome photographs from the Corel Image Database, portraying a variety of natural scenes. Each image was subjected to wavelet decomposition, using the $9 / 7$ symmetric biorthogonal wavelet basis function described by Antonini, Barlaud, Mathieu, and Daubechies (1992, p. 208; filter tap values given on p. 209). This resulted in a total of 13 sets of coefficients. Reconstructing the image from only the 1 st set of coefficients produces a very degraded (i.e., low-pass) version of the image; as more sets of coefficients are added, successively higher resolution versions are produced. Two versions of each picture were produced for the study, a high-resolution version that was reproduced by using all coefficients and a low-resolution version constructed by using only the first 4 sets of coefficients. Examples of these two versions of an image are shown in Figure 1.

\section{Apparatus}

The pictures were displayed in monochrome ( 8 bits per pixel) on a Conrac 9820T monitor under the control of a 386 computer, using a Kontrast model K8000 video card, in $512 \times 768$ pixel format, and refreshed at 144 frames per second, or about $7 \mathrm{msec}$ per frame. Image changes began at the millisecond at which they were requested, rather than waiting until the end of the current refresh cycle. The monitor was $89 \mathrm{~cm}$ from the participants' eyes, causing the picture to subtend $18^{\circ} \times 12^{\circ}$ of visual angle. The participants' eyes were tracked using a fifth-generation dual Purkinje image eyetracker, sampling the position of the right eye at $1000 \mathrm{~Hz}$ (i.e., once per millisecond). Viewing was binocular.

\section{Procedure}

The participants were shown the high-resolution version of each picture for $20 \mathrm{sec}$ as they prepared for a long-term change detection task. They then looked at each picture a second time, judging whether it was identical to or different from the picture presented earlier. Differences could include a mirror reversal (horizontal flip) or a replacement of one object in the picture with another. Prior to beginning the experiment, the participants were given practice in the task by viewing four example pictures and then four changed pictures, including examples of the above change types, as well as a change in camera angle.

As a participant examined the 36 experimental pictures, an online algorithm identif ied about every seventh saccade as being a critical saccade. Previous investigation had indicated that this frequency provided adequate time for a detection response to one experimental event before the next event was initiated (McConkie \& Currie, 1996). Subsequent off-line data analysis showed that $71 \%$ of the critical saccades occurred after seven intervening saccades, $24 \%$ occurred after five, six, or eight intervening saccades, and 5\% occurred after more. However, from the participant's point of view, it is clear that perceived critical fixations occurred with even less regularity, given that $18 \%$ of all critical saccades were controls involving no change (see below) and the overall detection rate of actual changes was $41 \%$. During each critical saccade, one of six conditions occurred. In the experimental conditions, as soon as the saccade was identified and approximately $8 \mathrm{msec}$ after the saccade actually began, the high-resolution image was replaced by its lowresolution version. Fourteen, 28, 42, 56, or $70 \mathrm{msec}$ later (close to even multiples of the frame rate), the high-resolution image returned to the screen. In the sixth condition, the control condition, no change occurred, leaving the picture in high-resolution form throughout the saccade. Each of the six conditions was scheduled to occur during the viewing of each picture, in a counterbalanced order across pictures and participants. During most trials, a participant was exposed to at least six experimental or control display changes, one for each of the six conditions, although the number of conditions that actually occurred depended, of course, on the number of saccades the participant made on that picture.

As a secondary task, the participants were asked to press a button if they noticed any flash, flicker, or blur occur in the picture as they examined it.

\section{RESULTS}

In analyzing the data, all critical saccades were first identified, excluding those cases in which the participant blinked, the display change algorithm failed to perform 

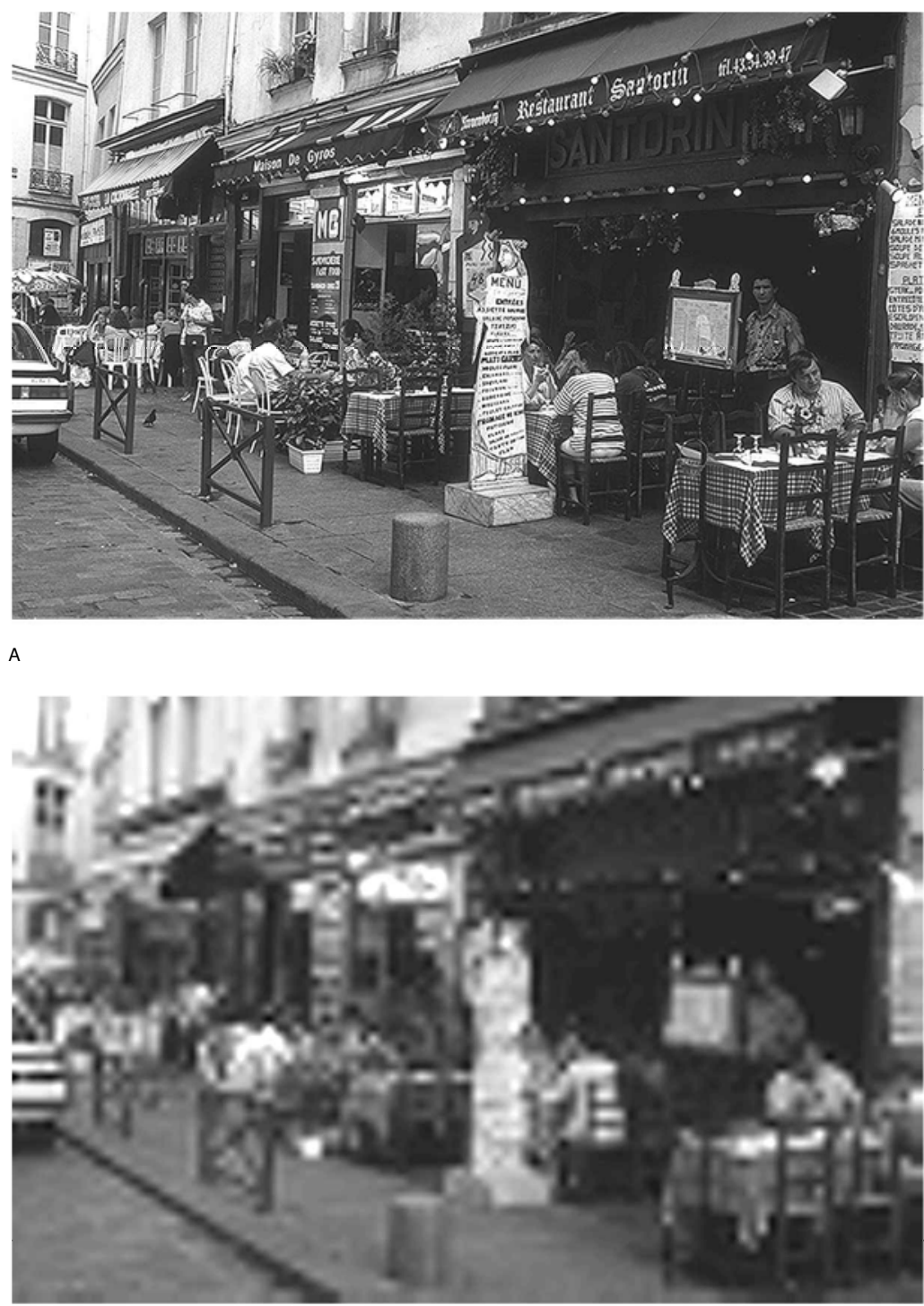

B

Figure 1. Example of a picture used in the study, including the normal (A) and the degraded (B) versions (normal image from the Corel Image Database).

as planned, or the picture ended before the seventh saccade after the critical saccade. Following these exclusions, 2,977 cases remained in the data set. Each of the remaining cases was coded by whether it was an experimental or a control condition case and by whether or not the participant pressed the button prior to the next critical saccade. Overall detection likelihood was $41 \%$, with only one false alarm $(0.2 \%$ false alarm rate). The correlation between trial number (1-36) and detection likelihood was small but significant $\left[r_{\mathrm{pb}}=.04 ; t(2,425)=\right.$ $2.16, p($ two-tailed $)=.03]$, indicating that, as one might predict, the participants' performance improved slightly with practice. However, when we removed the first five trials from the analysis, the correlation was no longer significant $\left[r_{\mathrm{pb}}=.02 ; t(2,046)=0.8, p(\right.$ two-tailed $)=$ $.424]$, suggesting that what improvements there were occurred rather quickly.

In addition, each case was coded by the velocity of the eyes at the change-back time, the change-back time relative to the peak of the overshoot in the Purkinje eyetracker signal, and the change-back time relative to the end of motion in the eyetracker signal. This information is illustrated in Figure 2. Velocity is represented as the amount of change in the eyetracker signal in a 4-msec period, beginning $2 \mathrm{msec}$ prior to and ending $2 \mathrm{msec}$ after the time at which the change-back was begun, which is then reported as degrees of visual angle. The eyetracker was adjusted so that $1^{\circ}$ of visual angle corre- 


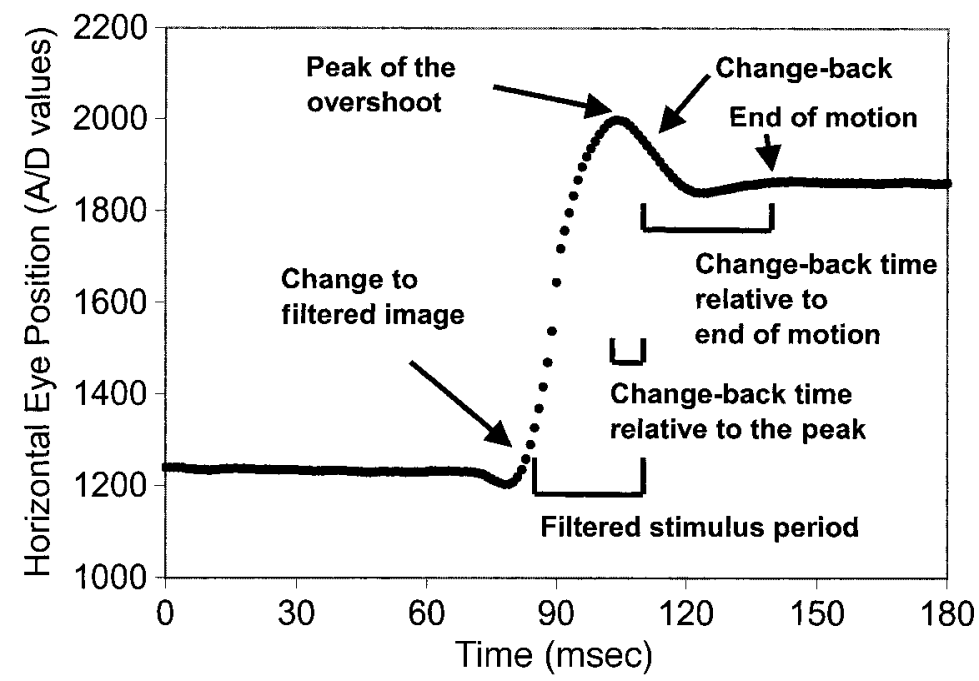

Figure 2. Horizontal component of a saccade made while a picture was viewed, showing the procedure used in the experiment. Sampling rate was $1000 \mathrm{~Hz}$. Critical events are marked: the millisecond at which the image was changed, the peak of the overshoot, the millisecond at which the change-back occurred, and the point at which motion ended. The period during which the filtered image was present on the screen is shown, as is the period from the peak to the change-back and from the end of motion to the change-back. In some cases, when the change-back occurred before the peak or the end of motion, a negative value resulted.

sponded to about 200 units change in the signal. The peak of the overshoot was identified as the sample on which the eye's distance from its position on the prior fixation was at a maximum. The end of eye motion was identified as the sample on which three difference measures $(x, y$, and the sum of the squared $x$ and $y$ differences) went below a change threshold of 4 analog units (or $4^{2}$ for the sum of squares measure), as compared with the sample 4 msec earlier.

\section{Detection as a Function of Change-Back Time Relative to the End of Motion of the Eyes}

Figure 3 shows the relationship between detection level, represented on the $y$-axis, and the time that the change-back occurred measured with the respect to the end of motion in the Purkinje eye movement signal, represented on the $x$-axis. As was indicated above, the end of motion probably indicates the time at which the motion of ocular structures-particularly, the lens-comes to an end. Negative values indicate change-back times occurring prior to the end of motion; positive values indicate change-back occurring after the end of motion. As Figure 3 indicates, $25-30 \mathrm{msec}$ before the end of motion, the detection level is about .22 and then rises steadily toward an asymptote that occurs at about the time the motion ends. These results clearly indicate that perception is occurring during the time that ocular motion continues, although the sensitivity is somewhat reduced. However, the results do not indicate a precise time at which perception begins.

\section{Detection as a Function of Change-Back Time Relative to the Peak of the Overshoot}

Figure 4 shows the relationship between detection level and the time that the change-back occurred, measured with respect to the peak of the overshoot in the Purkinje eye movement signal. As was indicated above, this peak corresponds with the time at which the surface of the eye stops moving (Deubel \& Bridgeman, 1995). Negative values indicate time prior to the peak of the overshoot, and positive values indicate time after the peak.

This figure shows a very strong relationship between change-back time and the likelihood of detection. Detection is essentially zero for change-back times prior to $6 \mathrm{msec}$ following the peak. At that point, detection begins a steep linear rise, with an asymptote at a high level. To further quantify this relationship, a three-segment linear model was fit to the original binary data, using a maximum likelihood loss function. Parameters indicate that during the first segment, detection is less than .01. Transition to the second segment occurs at $5.5 \mathrm{msec}$ following the peak. The slope of this segment is 0.03 , indicating this amount of increase in the detection level for every millisecond following the transition. The transition to the third, flat segment occurs $32 \mathrm{msec}$ after the peak, with a detection level of .80 .

It is possible that, even though the display change is not being consciously detected prior to $6 \mathrm{msec}$ after the peak, it still bothers the visual system when it occurs during that time. If so, there could be differences in the eye behavior data. To test for this possibility, a compar- 


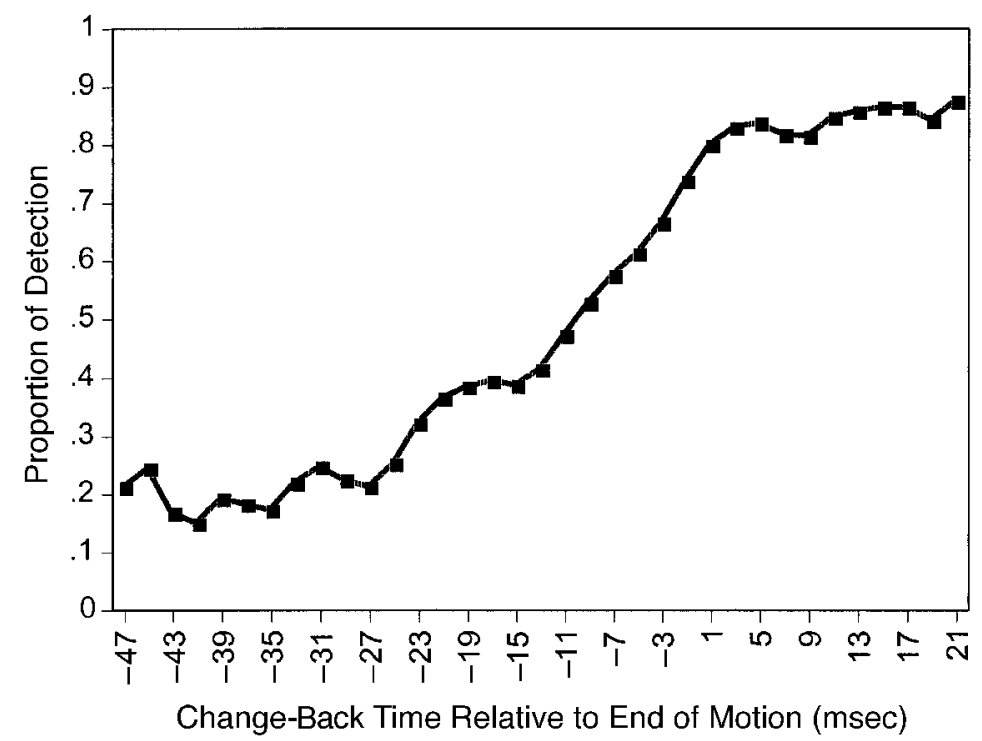

Figure 3. Change-back detection as a function of the time it occurred relative to the end of motion in the Purkinje eyetracker data. Detection frequencies were calculated for 2 -msec bins, and the data were then smoothed (average over a three-bin window, with the center bin weighted double).

ison was made between mean durations of fixations following saccades in the experimental (change) versus control (no change) conditions for cases in which the participants indicated no detection. The mean log fixation duration for each participant in each of the two con- ditions was computed, then compared across participants, using a paired $t$ test. No significant difference between conditions was found. In fact, the log fixation duration was slightly larger for the control condition, thus providing no support for the hypothesis that the visual

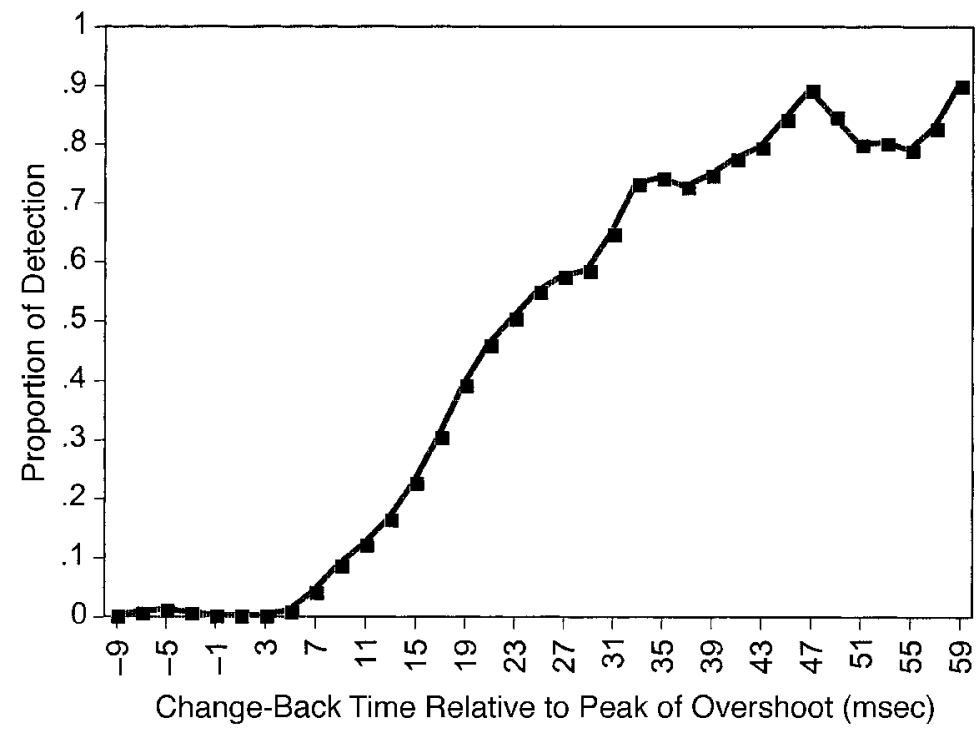

Figure 4. Change-back detection as a function of the time it occurred relative to the peak of the overshoot. Detection frequencies were calculated for 2 -msec bins, and the data were then smoothed (average over a three-bin window, with the center bin weighted double). 


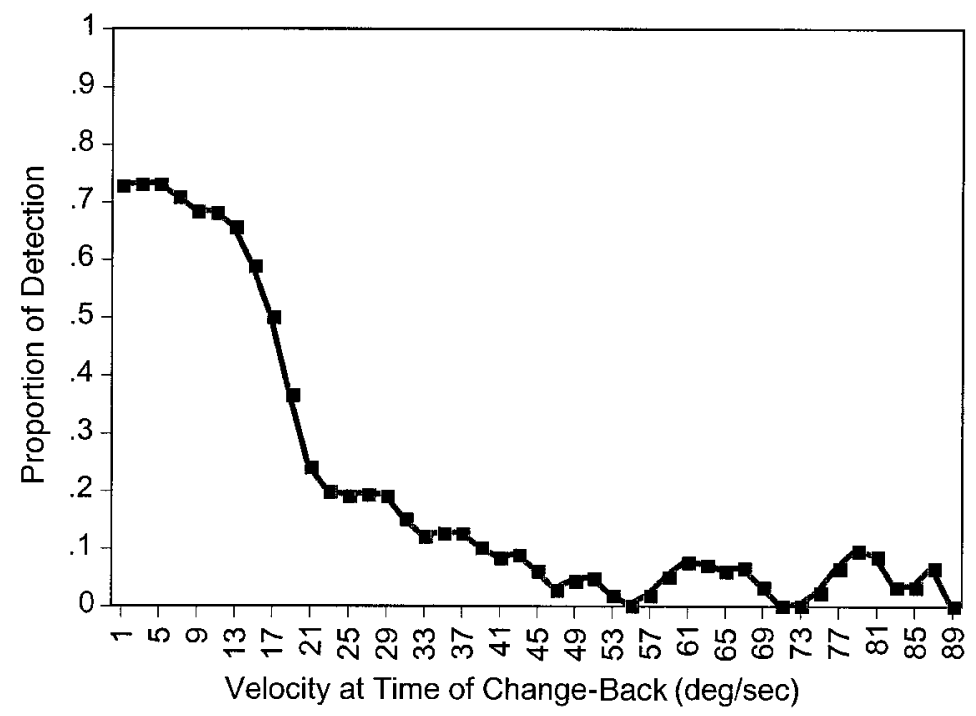

Figure 5. Change-back detection as a function of the velocity (in degrees/second) of the eyes at the time change-back occurred. Detection frequencies were calculated for $2-\mathrm{deg} / \mathrm{sec}$ bins, and the data were then smoothed (average over a three-bin window, with the center bin weighted double).

system is bothered by changes that are not consciously detected (experimental condition, $M=5.37$; control condition, $M=5.40)$.

\section{Detection as a Function of Velocity}

Figure 5 shows the relationship between eye velocity at the time of the change-back and the frequency with which that change was detected. Velocity is represented on the $x$-axis, presented in degrees of movement per second. The number of cases ranges from 110 to 235 per bin, with velocities in the 3-15 deg/sec range, down to $4-16$ for velocities over $50 \mathrm{deg} / \mathrm{sec}$.

Figure 5 indicates that detection likelihood was very low (but not zero) when the change-back occurred with saccade velocities greater than $45 \mathrm{deg} / \mathrm{sec}$; there were no detections when the velocity was greater than $90 \mathrm{deg} / \mathrm{sec}$. Detection rose linearly as velocity dropped from 45 to $24 \mathrm{deg} / \mathrm{sec}$ and then increased very rapidly as it dropped to $12 \mathrm{deg} / \mathrm{sec}$, at which point detection likelihood reached asymptote at just under .75 .

\section{DISCUSSION}

This study was conducted as an attempt to identify the perceptual onset time, or the time at which perception begins at the end of each saccade. The results are quite clear for the conditions studied: The availability of monochromatic, high spatial frequency information in a picture is not acquired sooner than $6 \mathrm{msec}$ after the peak of the overshoot, marking the end of motion of the eye's surface. The fact that there is still movement in the internal parts of the eye, as reflected in the Purkinje image eyetracker signal, does not appear to seriously delay the perceptual onset time. The low level of detection, shown in Figure 3, prior to the end of motion in the eyes appears to have resulted, not from the movement of internal structures, but instead from the fact that, in some cases, the change-back occurred prior to $6 \mathrm{msec}$ after the peak. This occurred less frequently at later times following the end of motion, causing the overall frequency of detection to rise.

Full, asymptotic perception develops by $32 \mathrm{msec}$ after the end of the saccade. Thus, if motion of internal eye structures reduces perception, it must occur during this period. Although detection likelihood is related to the velocity of the eye signal, this relation, for the Purkinje image eyetracker, appears to be due to its relationship with the time at which movement of the eye's surface ends. A closer relation might be found between velocity and detection if a search coil method of eyetracking, which is relatively unaffected by the motion of internal eye structures that appears to occur for $20 \mathrm{msec}$ or more after the actual end of the saccade, were to be used.

There is an important caveat to the conclusion that the perception onset time occurs about $6 \mathrm{msec}$ after the end of the saccade. In order for a person to detect a change in the display at a given moment, it is necessary to have acquired information from the previous display at some time prior to this. If it is assumed that the visual image does not survive the making of a saccade (Irwin, 1996; Irwin, Brown, \& Sun, 1988), this information must be obtained prior to the display change-back. Thus, in order to detect a change in the display made at $8 \mathrm{msec}$ after the end of the saccade, perception of the low spatial frequency image must have occurred prior to this time. Of course, we have no way of knowing just when that per- 
ception actually began. Thus, the above results indicate that perception actually begins prior to $6 \mathrm{msec}$ after the end of the saccade. By shortly after the 6-msec point, sufficient information has been obtained that the addition of higher frequency information to the picture can be detected or the retinal motion that accompanies this addition can be perceived. At present, a reasonable assumption is that perception begins at about the time that the eye stops rotating, at least for the types of display changes we made in the present study.

The results of this study confirm the fact that there is considerable suppression, or failure, of visual perception during the saccade (for a recent review, see Ross, Morrone, Goldberg, \& Burr, 2001). Removing most of the high spatial frequency information from the image and then returning the full information to the display is not detected so long as the change-back occurs within the first $5 \mathrm{msec}$ after the end of the saccade. This is consistent with observations, from a number of studies, that changes in text (McConkie \& Zola, 1979) and pictures (Currie et al., 2000; Grimes, 1996; Hayhoe et al., 1998; McConkie \& Currie, 1996; McConkie \& Loschky, 2000) that take place during saccades are not noticed. At the same time, some types of intrasaccadic display changes are detected. For example, Wolverton and Zola (1983) reported that removing text during saccades is very noticeable during reading. Current theory on saccadic suppression suggests that it strongly affects the magnocellular, but not the parvocellular, pathway, with low spatial frequency luminance modulated information and motion signals being strongly attenuated, but with little if any attenuation of chromatic information (Burr \& Morrone, 1996; Burr et al., 1994; Diamond et al., 2000; Ross et al., 2001; Shioiri \& Cavanagh, 1989). In sum, certain types of stimulus changes are perceived during saccades, and for other stimulus patterns that are not perceived during the saccade, perception onset time may still vary, with more perceptible changes being detected earlier. Further study is required to identify the amount of this variation. For experiments in which it is necessary to produce unperceived display changes during saccades, the method used in the present experiment can be employed to establish the perception onset time for the particular type of stimulus change being used, thus giving an indication of the time by which stimulus changes must be made in order to remain undetected.

An interesting question is whether failure to detect the change-back during the first $30 \mathrm{msec}$ of a fixation may actually result from the high-resolution image's masking the emerging perception of the low-resolution image early in the fixation. Two general theories of visual masking explain the phenomenon in terms of interruption of processing by the mask or integration with the mask (Felsten \& Wasserman, 1980). Explaining change-back detection failure on the basis of interruptive masking requires arguing that the spatial transient associated with the changeback interrupts processing of the low-resolution image, while simultaneously arguing that the transient itself is imperceptible. An integrative masking explanation, in which it is argued that the emerging perception of the low spatial frequencies in the degraded image become integrated with the perception of the wider band of spatial frequencies contained in the change-back image, seems more plausible. Given that low spatial frequencies tend to be processed earlier than high spatial frequencies (Schyns \& Oliva, 1993; Vassilev \& Stomonyakov, 1987), with an early enough change-back, the integration of lower and higher spatial frequency information in the two image versions might be seamless. Therefore, integrative masking of the change image by the change-back image might explain part of the postsaccadic suppression observed in our study. If so, such masking effects appear to be rapidly weakened as the perceptual representation of lower spatial frequencies in the degraded image evolve early during the fixation, so that detection of the changeback occurs on the basis of perceiving either the lack of higher spatial frequencies in the change image or the addition of higher spatial frequencies contained in the change-back image. Further studies may be needed to investigate this issue.

There is a linear increase in detection likelihood over the $25 \mathrm{msec}$ after the onset of perception. Although this is quite a short growth period, it should be noted that it is probably still an overestimation. Since the present study was conducted with a cathode-ray tube display, the actual time after the saccade at which the image is being painted onto that part of the computer screen to which the gaze is directed is somewhat variable. With a 7-msec frame rate, about this amount of variation must exist, from fixation to fixation, in when the part of the image to which the gaze is directed is overwritten with the new version of the image. Second, although Deubel and Bridgeman (1995) argued that the peak of the overshoot in the dual Purkinje image eyetracker occurs at about the same time that the search coil indicates the end of the external eye motion, this correspondence is probably not exact. If it is the case that the end of the saccade is truly the earliest perception onset time, these two sources of variability probably reduce the slope with which detection likelihood increases over time. Thus, the rise of perception of high spatial frequency information appears to be very rapid after the end of the saccade.

Whether or not a display change that occurs at the end of a saccade will be detected can also be predicted on the basis of the velocity of the eyes at the time that the change is made. Changing the image at the time of a $40 \mathrm{deg} / \mathrm{sec}$ velocity or greater, using the Purkinje image eyetracker, results in a detection rate below .10, but velocities over $90 \mathrm{deg} / \mathrm{sec}$ are required to reach zero response levels. Consequently, it appears that when a near zero detection rate is needed, the use of a time criterion (initiating the change before $6 \mathrm{msec}$ after the completion of the saccade) is probably more efficient than the use of a velocity criterion. However, this result may be specific to the Purkinje image eyetracker, since it does not give an accurate representation of the eyes' velocity during a 
saccade, overestimating that velocity in the latter part of the saccade (Deubel \& Bridgeman, 1995). Further research is needed in order to determine whether the time and velocity measures can be combined in order to more effectively predict whether, or with what frequency, a display change will be detected.

Numerous studies of saccadic suppression have shown that it continues beyond the end of a saccade for a period of $50 \mathrm{msec}$ or more (Burr et al., 1994; Diamond et al., 2000; Matin, 1974; Shioiri \& Cavanagh, 1989; Volkmann, 1962; Volkmann et al., 1978). The observation that, in the present study, image change detection began to occur at or perhaps before $6 \mathrm{msec}$ and reached full sensitivity within roughly $30 \mathrm{msec}$ of the completion of the saccade is consistent with these earlier findings. However, for practical purposes, it suggests that this suppression is relatively mild, and if one's criterion is seamless perception of a gaze-contingent display, it offers only a small window of time in which to change the image.

A related issue concerns what deadline to use for updating the display area of interest in a gaze-contingent multiresolutional display (GCMRD; Reingold et al., in press). In our studies of the perceptual effects of GCMRDs (Loschky \& McConkie, 2000, 2002; Loschky et al., 2002), we have used a deadline of $5 \mathrm{msec}$ after the peak of the overshoot, on the basis of the results of the present experiment. This was done to ensure that any effects on perception would not be due to detection of the motion transient caused by the display change but, rather, to the other variables we manipulated, such as the drop-off of image resolution with retinal eccentricity. However, it should be noted that the present experiment did not make image changes of the type that would normally occur in a GCMRD. In the present experiment, our change was from a constant high-resolution image to a constant lowresolution image, and the change-back was vice versa. In contrast, in a GCMRD, there would normally be only one change, from an image with high resolution at the location of the preceding center of gaze and lower resolution elsewhere to an image with high resolution at the current center of gaze and lower resolution elsewhere. Thus, in order to determine the latest possible deadline for updating a GCMRD without affecting perception, we are conducting further studies using multiresolutional images of the type used in GCMRDs.

The results from this experiment are also relevant to another issue mentioned earlier: At what time should the clock be started for measuring fixation durations and online stimulus display intervals? The results indicate that plotting detection likelihood against time relative to when the eye stops rotating (i.e., peak of the overshoot in the Purkinje eyetracker signal) gives a much steeper function than plotting it against time relative to when internal parts of the eyes stop moving (i.e., end of motion in the Purkinje eyetracker signal). This argues that the onset of perception can best be defined in relation to the end of rotational motion of the eyes. This gives a psychological justification for measuring fixation time from the end of the preceding saccade, rather than just an oculomotor justification: The perception of the stimulus present on a fixation apparently begins around that time. There is no significant delay owing to saccadic suppression or to movement in the internal structures of the eyes. It also suggests that researchers using dual Purkinje image eyetrackers should probably use the peak of the overshoot as the onset point for measuring fixation durations and brief saccade-linked stimulus onset periods. Since many laboratories in which these eyetrackers have been used for studying reading and other cognitive tasks, including our own (McConkie, Zola, Wolverton, \& Burns, 1978), have measured time from the point at which eye motion falls below a low criterion, which usually occurs some time after the peak of the overshoot, following this recommendation would increase mean eye fixation durations in these studies, probably by $20 \mathrm{msec}$ or more. An advantage of adopting the peak of the overshoot as the point from which to measure time in a fixation, besides the psychological justification given above, is that this is a landmark in the data that is relatively unambiguous. Adopting this standard should result in greater data consistency in the identification of fixation onset times and of fixation durations across laboratories.

Finally, it must be recognized that the definition given above for the concept of perception onset time is a very conservative one; it is taken as the point at which the initiation of a display change begins to increase detection responses above the baseline detection when no changes occur or when they occur during the saccade, both of which were approximately zero in this study. At that point, detection is rare but occurs in some cases. This is a useful benchmark when research is conducted in which display changes must be made before any possibility of detection. For theoretical purposes, it may be more appropriate to define the perception onset time as the point in time at which the detection level reaches halfway to the asymptote (by this time detection has occurred in half the cases in which it will eventually occur) or even as the time at which the asymptote is reached (the point in time at which this aspect of perception is fully developed). These definitions will produce later perception onset time estimates (i.e., 22 or $38 \mathrm{msec}$ in the present case) and represent different meanings of the term. It may also be appropriate to increase these values further by adding time related to the display's refresh rate, in an attempt to define when it is that visual perception should be said to begin after a saccade.

\section{REFERENCES}

Antonini, M., Barlaud, M., Mathieu, P., \& Daubechies, I. (1992). Image coding using wavelet transform. IEEE Transactions on Image Processing, 1, 205-220.

Bertera, J. H., \& RAYNer, K. (2000). Eye movements and the span of the effective stimulus in visual search. Perception \& Psychophysics, 62, 576-585.

Brooks, B. A., Impelman, D. M. K., \& Lum, J. T. (1981). Backward and forward masking associated with saccadic eye movement. Perception \& Psychophysics, 30, 62-70.

Burr, D. C., \& Morrone, M. C. (1996). Temporal impulse response 
functions for luminance and colour during saccades. Vision Research, 36, 2069-2078.

Burr, D. C., Morrone, M. C., \& Ross, J. (1994). Selective suppression of the magnocellular visual pathway during saccadic eye movements. Nature, 371, 511-513.

CheKaluk, E., \& Llewellyn, K. R. (1990). Visual stimulus input, saccadic suppression, and detection of information from the postsaccade scene. Perception \& Psychophysics, 48, 135-142.

Chekaluk, E., \& Llewellyn, K. R. (1994). Masking effects in saccadic eye movements. In G. d'Ydewalle \& J. Van Rensbergen (Eds.), Visual and oculomotor functions: Advances in eye movement research (Vol. 5, pp. 45-54). Amsterdam: Elsevier, North-Holland.

Collewijn, H., Van der Mark, F., \& Jansen, T. C. (1975). Precise recording of human eye movements. Vision Research, 15, 447-450

Crane, H. D., \& Steele, C. S. (1978). Accurate three-dimensional eyetracker. Applied Optics, 17, 691-705.

Currie, C. B., McConkie, G. W., Carlson-Radvansky, L. A. \& Irwin, D. E. (2000). The role of the saccade target object in the perception of a visually stable world. Perception \& Psychophysics, 62, 673-683.

Deubel, H., \& BRidgeman, B. (1995). Fourth Purkinje image signals reveal eye-lens deviations and retinal image distortions during saccades. Vision Research, 35, 529-538.

Diamond, M. R., Ross, J., \& Morrone, M. C. (2000). Extraretinal control of saccadic suppression. Journal of Neuroscience, 20, 34493455 .

Felsten, G., \& Wasserman, G. S. (1980). Visual masking: Mechanisms and theories. Psychological Bulletin, 88, 329-353.

GRImES, J. (1996). On the failure to detect changes in scenes across saccades. In K. A. Akins (Ed.), Perception (Vol. 5, pp. 89-110). New York: Oxford University Press.

Hay hoe, M. M., Bensinger, D. G., \& Ballard, D. H. (1998). Task constraints in visual working memory. Vision Research, 38, 125-137.

Henderson, J. M., \& Hollingworth, A. (1999). The role of fixation position in detecting scene changes across saccades. Psychological Science, 10, 438-443.

Henderson, J. M., McClure, K. K., Pierce, S., \& Schrock, G. (1997). Object identification without foveal vision: Evidence from an artificial scotoma paradigm. Perception \& Psychophysics, 59, 323-346.

IRWIN, D. E. (1996). Integrating information across saccadic eye movements. Current Directions in Psychological Science, 5, 94-100.

IrwIN, D. E., Brown, J. S., \& SuN, J.-S. (1988). Visual masking and visual integration across saccadic eye movements. Journal of Experimental Psychology: General, 117, 276-287.

LoschKy, L. C., \& McConkie, G. W. (2000). User performance with gaze contingent multiresolutional displays. In A. T. Duchowski (Ed.), Proceedings of the Eye Tracking Research \& Applications Symposium 2000 (pp. 97-103). New York: ACM Press.

Loschky, L. C., \& McConkie, G. W. (2002). Investigating spatial vision and dynamic attentional selection using a gaze-contingent multiresolutional display. Journal of Experimental Psychology: Applied, 8 99-117.

Loschky, L. C., McConkie, G. W., YAng, J., \& Miller, M. E. (2002). The effects of eccentricity-dependent image filtering on saccade targeting in natural images [Abstract]. Journal of Vision, 2(7), 170a. http://journal ofvision.org/2/7/170/, DOI 10.1167/2.7.170.

Matin, E. (1974). Saccadic suppression: A review and an analysis. Psychological Bulletin, 81, 899-917.

McConkie, G. W., \& Currie, C. B. (1996). Visual stability across saccades while viewing complex pictures. Journal of Experimental Psychology: Human Perception \& Performance, 22, 563-581.

McConkie, G. W., \& Loschky, L. C. (2000, Month). Failure to detect intrasaccadic object location changes in pictures. Paper presented at the Annual Conference of the Midwest Psychological Association, Chicago, IL.

McConkie, G. W., \& Rayner, K. (1975). The span of the effective stimulus during a fixation in reading. Perception \& Psychophysics, 17, 578-586.
McConkie, G. W., \& ZoLA, D. (1979). Is visual information integrated across successive fixations in reading? Perception \& Psychophysics, 25, 221-224.

McConkie, G. W., Zola, D., Wolverton, G. S., \& Burns, D. D. (1978). Eye movement contingent display control in studying reading. Behavior Research Methods \& Instrumentation, 10, 154-166.

Morrison, R. E. (1984). Manipulation of stimulus onset delay in reading: Evidence for parallel programming of saccades. Journal of Experimental Psychology: Human Perception \& Performance, 10, 667682.

Paus, T., Marrett, S., Worsley, K. J., \& Evans, A. C. (1995). Extraretinal modulation of cerebral blood flow in the human visual cortex Implications for saccadic suppression. Journal of Neurophysiology, 74, 2179-2183

RAYNER, K. (1997). Understanding eye movements in reading. Scientific Studies of Reading, 1, 317-339.

Rayner, K., Inhoff, A. W., Morrison, R. E., Slowiaczek, M. L., \& BerterA, J. H. (1981). Masking of foveal and parafoveal vision during eye fixations in reading. Journal of Experimental Psychology: Human Perception \& Performance, 7, 167-179.

Reingold, E. M., Loschky, L. C., McConkie, G. W., \& Stampe, D. M. (in press). Gaze-contingent multi-resolutional displays: An integrative review. Human Factors.

Reingold, E. M., \& Stampe, D. M. (1999). Saccadic inhibition in complex visual tasks. In W. Becker, H. Deubel, \& T. Mergner (Eds.), Current oculomotor research: Physiological and psychological aspects (pp. 249-255). London: Plenum.

Ross, J., Morrone, M. C., Goldberg, M. E., \& Burr, D. C. (2001). Changes in visual perception at the time of saccades. Trends in Neurosciences, 24, 113-121.

Schyns, P., \& Oliva, A. (1993). From blobs to boundary edges: Evidence for time- and spatial-scale-dependent scene recognition. Psychological Science, 5, 195-200.

ShIOIRI, S. (1993). Postsaccadic processing of the retinal image during picture scanning. Perception \& Psychophysics, 53, 305-314.

Shioiri, S., \& CaVAnagh, P. (1989). Saccadic suppression of low-level motion. Vision Research, 29, 915-928.

UCHIKAWA, K., \& SATo, M. (1995). Saccadic suppression of achromatic and chromatic responses measured by increment-threshold spectral sensitivity. Journal of the Optical Society of America, 12, 661-666.

van Diepen, P. M. J., Ruelens, L., \& D'Ydewalle, G. (1999). Brief foveal masking during scene perception. Acta Psychologica, 101, 91103.

VAN DiePen, P. M. J., \& WAMPERs, M. (1998). Scene exploration with Fourier-filtered peripheral information. Perception, 27, 1141-1151. van Diepen, P. M. J., Wampers, M., \& D'Ydewalle, G. (1995, September). The use of coarse and fine peripheral information during scene perception. Paper presented at the Eighth European Conference on Eye Movements, Derby, U.K.

van Diepen, P. M. J., Wampers, M., \& D'Ydewalle, G. (1998). Functional division of the visual field: Moving masks and moving windows. In G. Underwood (Ed.), Eye guidance in reading and scene perception (pp. 337-355). Oxford: Anonima Romana.

VASSILEV, A., \& STOMONyAKov, V. (1987). The effect of grating spatial frequency on the early VEP-component CI. Vision Research, 27, 727729

Volkmann, F. C. (1962). Vision during voluntary saccadic eye movements. Journal of the Optical Society of America, 52, 571-578.

Volkmann, F. C., Riggs, L. A., White, K. D., \& Moore, R. K. (1978). Contrast sensitivity during saccadic eye movements. Vision Research, 18, 1193-1199.

Wolverton, G. S., \& Zola, D. (1983). The temporal characteristics of visual information extraction during reading. In K. Rayner (Ed.), Eye movements in reading: Perceptual and language processes (pp. 4151). New York: Academic Press.

(Manuscript received March 20, 2002; revision accepted for publication October 21, 2002.) 